$05,10,11$

\title{
Декорированная изинговская квадратная решетка в магнитном поле
}

\author{
(C) Ф.А. Кассан-Оглы ${ }^{1}$, А.И. Прошкин ${ }^{1,2}$, А.К. Муртазаев $^{3}$, В.А. Мутайламов ${ }^{3}$ \\ ${ }^{1}$ Институт фризики металлов им. М.Н. Михеева УрО РАН, \\ Екатеринбург, Россия \\ ${ }^{2}$ Уральский федеральный университет им. Б.Н. Ельцина, \\ Екатеринбург, Россия \\ ${ }^{3}$ Институт фризики им. Х.И. Амирханова ДФИЦ РАН, \\ Махачкала, Россия \\ E-mail: felix.kassan-ogly@imp.uran.ru
}

Поступила в Редакцию 30 декабря 2019 г.

В окончательной редакции 30 декабря 2019 г.

Принята к публикации 10 января 2020 г.

\begin{abstract}
Исследована модель Изинга на декорированной квадратной решетке с различными параметрами обменных взаимодействий во внешнем магнитном поле. Проведено сравнение с численными результатами, полученными для модели Изинга на простой квадратной решетке. Показано, что если намагниченность при увеличении поля возрастает по антиферромагнитному типу, то происходит плавное уменьшение точки исходного фазового перехода до нуля при достижении первого фрустрационного поля, и при дальнейшем увеличении поля фазовый переход не возникает. В случае возрастания намагниченности по ферромагнитному типу, фазовый переход исчезает сразу же при включении поля, то есть сколь угодно малое магнитное поле полностью подавляет фазовый переход.
\end{abstract}

Ключевые слова: модель Изинга, декорированная квадратная решетка, магнитное поле, фрустрации.

DOI: $10.21883 /$ FTT.2020.05.49230.20M

\section{1. Введение}

За последние годы значительно возрос интерес к исследованию так называемых декорированных решеток благодаря разнообразию наблюдаемых в них новых явлений и особенностей по сравнению с исходными (недекорированными) решетками. В частности, декорирование порождает множество фрустрационных эффектов, оно может приводить как к подавлению фазовых переходов, существующих в недекорированных решетках, так и к возникновению новых фазовых переходов, отсутствующих в исходных решетках, в том числе и множественных переходов. Кроме того, появляются новые типы частичного упорядочения, а также разнообразные формы расщепления теплоемкости. Богатство критического поведения декорированных решеток обусловлено возможной многократностью декорирования.

Понятие декорированная решетка, относящееся к магнитной модели Изинга, впервые предложено в 1951 г. в работе Сиози [1]. Суть его заключается во введение дополнительных спинов в промежутки между узлами исходной решетки. Это понятие можно обобщить и на кристаллические решетки. Например, ОЦКи ГЦК-решетки можно воспринимать, как кубическая объемно-декорированная и кубическая гранедекорированная. В данном случае декорирование приводит к хорошо известному существенному эффекту - эффекту экстинкции, а именно, происходит исчезновение половины рефлексов [2]. Фактически, подавляющее большинство реальных структур являются декорированными, делая тем самым исследование влияние декорирования насущным и весьма плодотворным.

Целью данной работы было исследование влияния внешнего магнитного поля на термодинамические и магнитные свойства декорированной квадратной решетки в модели Изинга и сравнение с исходной (недекорированной) решеткой.

\section{2. Основные формулы и методы расчета}

Гамильтониан модели Изинга на квадратной решетке с любыми кратностями декорирования по направлениям $x$ и $y$ с учетом обменных взаимодействий (прямых взаимодействий) между спинами в исходной решетке (нодальными спинами), между декорационными и нодальными спинами, между самими декорационными спинами, а также и при наличии внешнего магнитного поля записывается в виде

$$
\begin{aligned}
& \mathscr{H}=-\sum_{x=1}^{N_{x}} \sum_{y=1}^{N_{y}}\left(J_{x} \sigma_{x, y} \sigma_{x+1, y}+J_{y} \sigma_{x, y} \sigma_{x, y+1}\right) \\
& -\sum_{x=1}^{N_{x}} \sum_{y=1}^{N_{y}}\left(\sum_{i=0}^{d_{x}} J_{x}^{\prime} \sigma_{x+i, y} \sigma_{x+i+1, y}+\sum_{j=0}^{d_{y}} J_{y}^{\prime} \sigma_{x, y+j} \sigma_{x, y+j+1}\right) \\
& -\sum_{n=1}^{N_{x} N_{y} Z} h \sigma_{n}
\end{aligned}
$$

где $J_{x}$ и $J_{y}-$ взаимодействия между нодальными спинами, $J_{x}^{\prime}$ и $J_{y}^{\prime}$ - взаимодействия между декораци- 


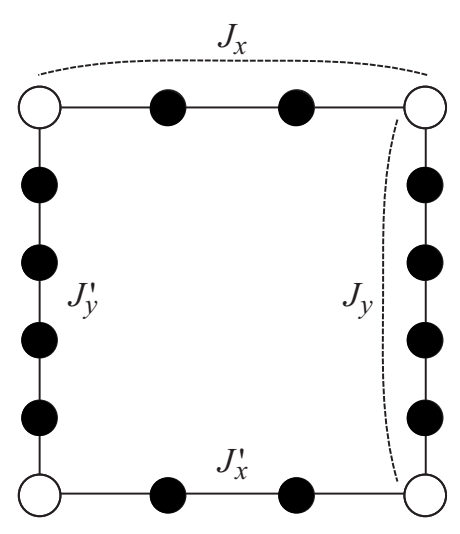

Рис. 1. Декорированная квадратная решетка.

онными и нодальными спинами совпадают с взаимодействиями между самими декорационными спинами, $h$ - внешнее магнитное поле, все операторы спина $\sigma= \pm 1, Z=\left(1+d_{x}+d_{y}\right)-$ число спинов в элементарной ячейке. Рис. 1 представляет в качестве примера элементарную ячейку квадратной решетки, дважды декорированной по направлению $x$ и четырехкратно декорированной по направлению $y$.

Следуя идеологии Сиози [1] и используя предложенное им декорационно-итерационное преобразование (decoration-iteration transformation), мы вывели общее аналитическое выражение для максимального значения трансфер-матрицы Крамерса-Ваннье (2) в рассматриваемой модели с произвольной кратностью декорирования по направлениям $x$ и $y$.

Следует отметить, что декорационно-итерационное преобразование Сиози не позволяет задавать разные значения для обменных взаимодействий между декорационными и нодальными спинами и взаимодействиями между самими декорационными спинами. Кроме того, аналитического решения для декорированных решеток в магнитном поле даже с использованием декорационноитерационного преобразования, впрочем, также как и для исходных (недекорированных) решеток, до сих пор не получено.

$$
\begin{aligned}
& \ln \frac{\lambda}{2}=\frac{1}{2 \pi^{2} Z} \\
& \quad \times \int_{0}^{\pi} \ln \left(C_{x} C_{y}-D_{y} S_{x} \cos \phi-D_{x} S_{y} \cos \theta\right) d \phi d \theta,
\end{aligned}
$$

где $d_{x}$ и $d_{y}$ - это кратности декорирования по направлениям $x$ и $y$,

$$
\begin{gathered}
D_{x}=\cosh \left(\frac{J_{x}^{\prime}}{T}\right)^{2 d_{x}+2}-\sinh \left(\frac{J_{x}^{\prime}}{T}\right)^{2 d_{x}+2}, \\
C_{x}=\frac{1}{2} \exp \left(2 J_{x} / T\right)\left(\cosh \left(\frac{J_{x}^{\prime}}{T}\right)^{d_{x}+1}+\sinh \left(\frac{J_{x}^{\prime}}{T}\right)^{d_{x}+1}\right)^{2} \\
+\frac{1}{2} \exp \left(-2 J_{x} / T\right)\left(\cosh \left(\frac{J_{x}^{\prime}}{T}\right)^{d_{x}+1}-\sinh \left(\frac{J_{x}^{\prime}}{T}\right)^{d_{x}+1}\right)^{2},
\end{gathered}
$$

$$
\begin{aligned}
& S_{x}=\frac{1}{2} \exp \left(2 J_{x} / T\right)\left(\cosh \left(\frac{J_{x}^{\prime}}{T}\right)^{d_{x}+1}+\sinh \left(\frac{J_{x}^{\prime}}{T}\right)^{d_{x}+1}\right)^{2} \\
& -\frac{1}{2} \exp \left(-2 J_{x} / T\right)\left(\cosh \left(\frac{J_{x}^{\prime}}{T}\right)^{d_{x}+1}-\sinh \left(\frac{J_{x}^{\prime}}{T}\right)^{d_{x}+1}\right)^{2}
\end{aligned}
$$

Для получения базовых результатов (в отсутствие поля) мы использовали аналитические выражения (2)-(5), а также формулы, явно выражающие энтропию $S$, теплоемкость $C$ и параметр порядка $\eta$, введенный в работе [3], через максимальное значение трансфер-матрицы Крамерса-Ваннье $\lambda$ :

$$
\begin{gathered}
S=\ln \lambda+\frac{T}{\lambda} \frac{\partial \lambda}{\partial T}, \\
C=\frac{2 T}{\lambda} \frac{\partial \lambda}{\partial T}+\frac{T^{2}}{\lambda} \frac{\partial^{2} \lambda}{\partial T^{2}}-\frac{T^{2}}{\lambda^{2}}\left(\frac{\partial \lambda}{\partial T}\right)^{2}, \\
\eta=1-\frac{\ln \lambda}{\ln 2}-\frac{T}{\lambda \ln 2} \frac{\partial \lambda}{\partial T} .
\end{gathered}
$$

При наличии внешнего магнитного поля расчеты всех наблюдаемых выполнялись методом компьютерного моделирования, используя наиболее эффективный алгоритм Ванга-Ландау [4,5].

\section{3. Влияние поля на недекорированной квадратной решетке в модели Изинга}

Исследование модели Изинга на квадратной решетке во внешнем магнитном поле привлекало внимание сразу после получения Онзагером знаменательного точного решения в отсутствие поля [6].

Первым удачным результатом решения этой интригующей задачи послужила работа Мюллера-Гартмана и Циттарса [7], в которой буквально в качестве догадки они предложили формулу зависимости температуры фазового перехода от поля:

$$
\cosh \left(\frac{h}{T_{c}}\right)=\sinh \left(\frac{2 J_{x}}{T_{c}}\right) \sinh \left(\frac{2 J_{y}}{T_{c}}\right) .
$$

Впоследствии эта зависимость многократно проверялась и уточнялась разнообразными численными расчетами, в том числе и нашими расчетами теплоемкости при нескольких значениях магнитного поля, причем расхождение возникает лишь в четвертом значащем знаке. Однако следует отметить ограниченную применимость этой формулы только для антиферромагнитных обменных взаимодействий $J_{x}<0$ и $J_{y}<0$. Магнитные и фрустрационные свойства антиферромагнитной модели Изинга на недекорированной решетке исследованы в статье [8]. На рис. 2 представлена намагниченность недекорированной антиферромагнитной модели Изинга, как функции поля, так и температуры.

Мы выполнили расчеты несколько вариантов численных значений $J_{x}$ и $J_{y}$, в том числе и с разными знаками 

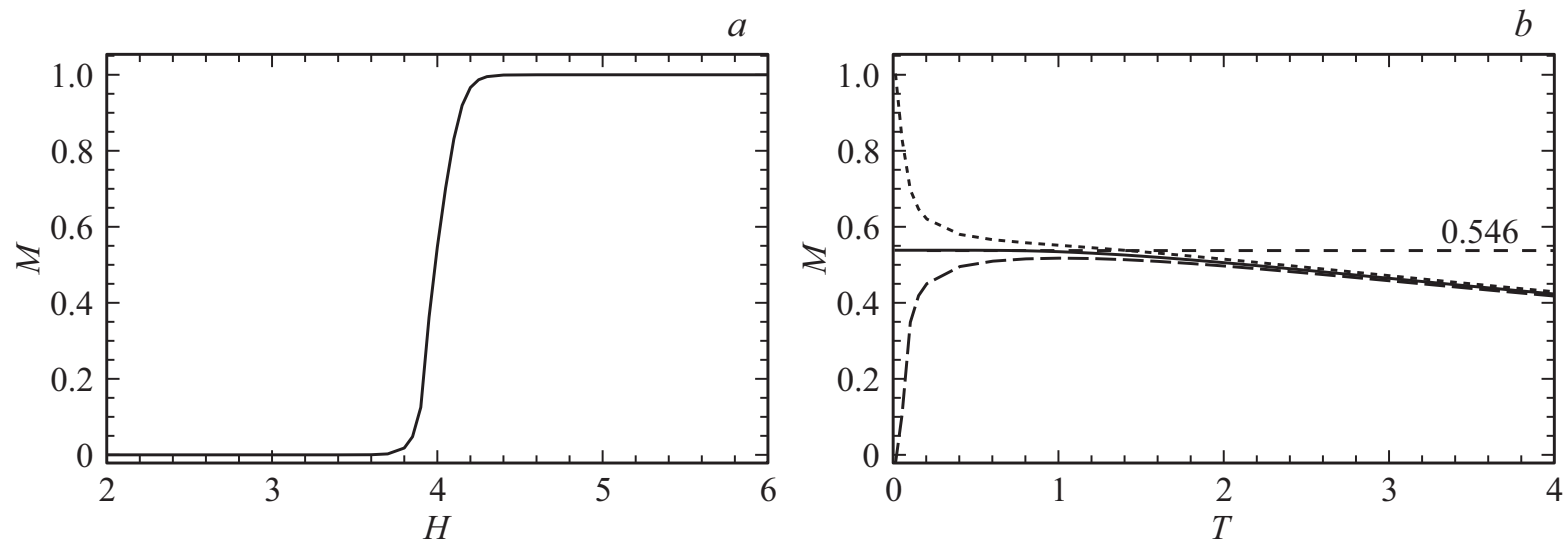

Рис. 2. Антиферромагнитная модель Изинга на недекорированной квадратной решетке $\left(J_{x}=J_{y}=-1\right)$ в магнитном поле: (a) - намагниченность при $T=0.1 ;(b)-h=3.95$ (штриховая линия), $h=4.0$ (сплошная линия), $h=4.05$ (пунктирная линия).
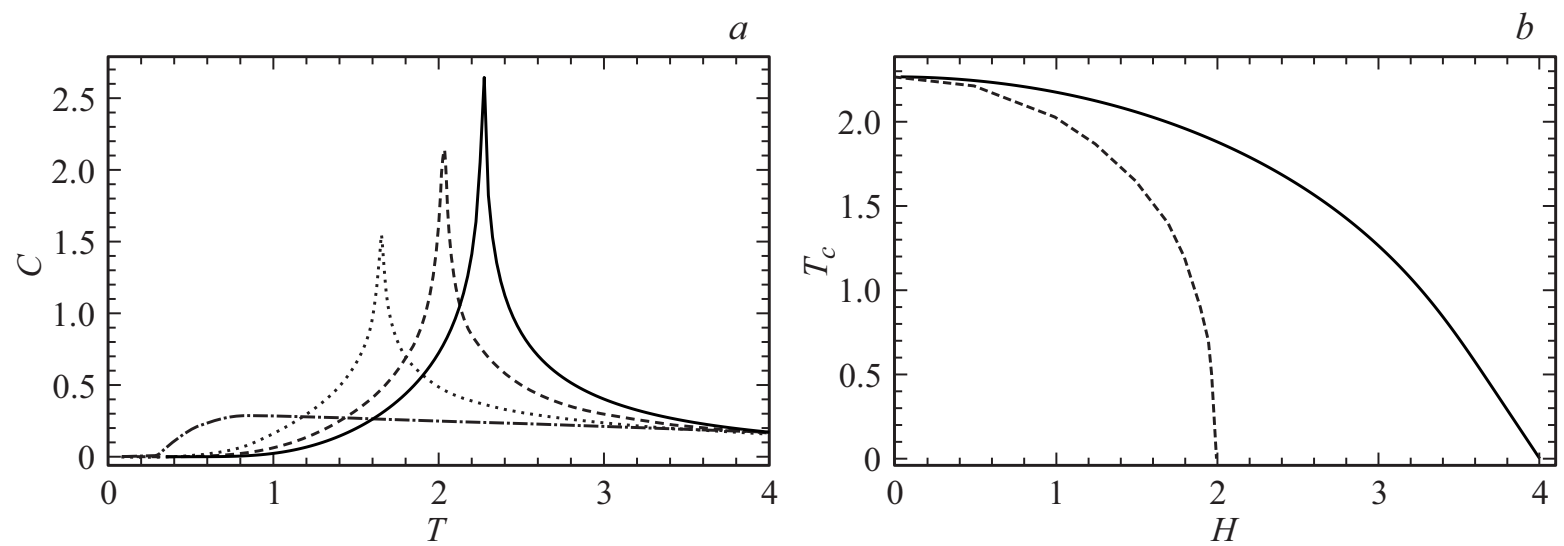

Рис. 3. Ферро-антиферромагнитная модель Изинга на квадратной решетке $\left(J_{x}=1, J_{y}=-1\right)$ в магнитном поле: $(a)-$ теплоемкость при $h=0$ (сплошная линия), $h=1$ (штриховая), $h=1,5$ (пунктирная), $h=2$ (штрих-пунктирная); $(b)-$ зависимость температуры перехода от приложенного поля (сплошная линия). Штриховая — температура перехода при $J_{x}=-1, J_{y}=-1$.
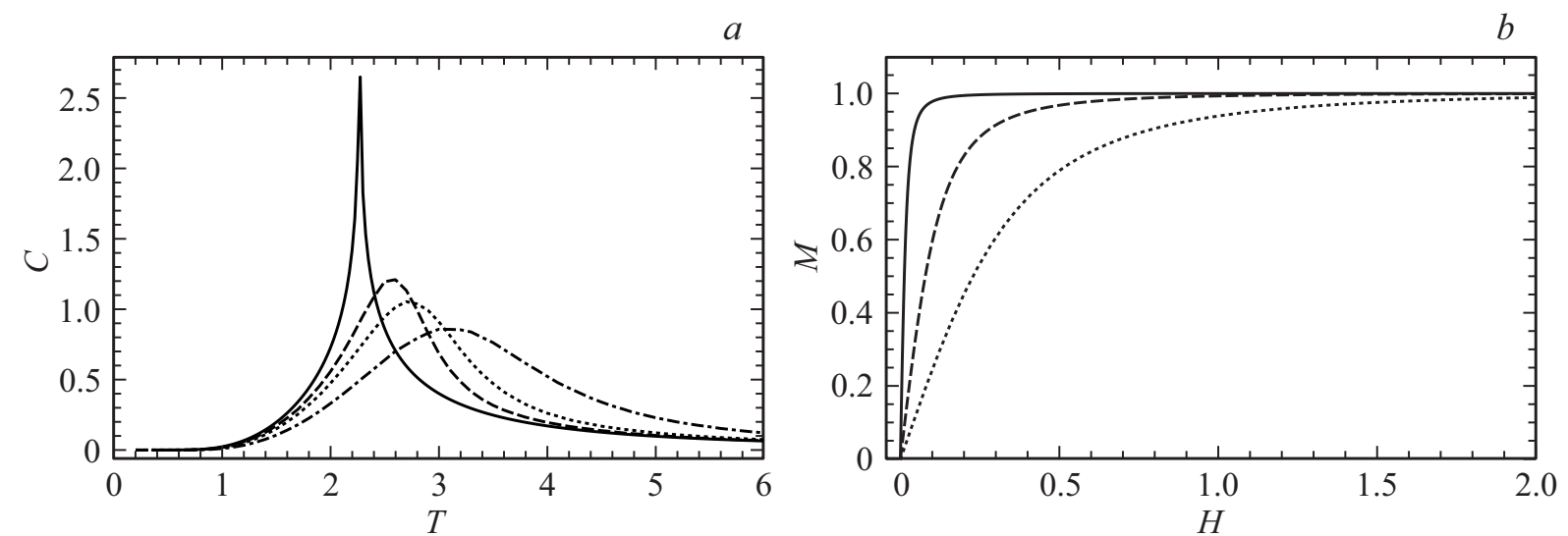

Рис. 4. Ферромагнитная модель Изинга на квадратной решетке $\left(J_{x}=J_{y}=1\right)$ в магнитном поле: $(a)-$ теплоемкость при $h=0$ (сплошная линия), $h=0.1$ (штриховая), $h=0.2$ (пунктирная), $h=0.5$ (штрих-пунктирная); $(b)-$ намагниченность при $T=0.3$ (сплошная линия), $T=0.5$ (штриховая), $T=1.1$ (пунктирная).

$J_{x}<0$ и $J_{y}>0$. На рис. 3, $a$ изображена зависимость теплоемкости от температуры для нескольких значений поля при $J_{x}=+1$ и $J_{y}=-1$, а на рис. $3, b$ результирующая кривая зависимости температуры перехода от поля (сплошная линия), причем для сравнения приведена рассчитанная зависимость по формуле Мюллера-Гартмана и Циттарса (9) при $J_{x}=+1$ и $J_{y}=+1$. Из рис. 3 непригодность формулы (9) для разных знаков взаи- 

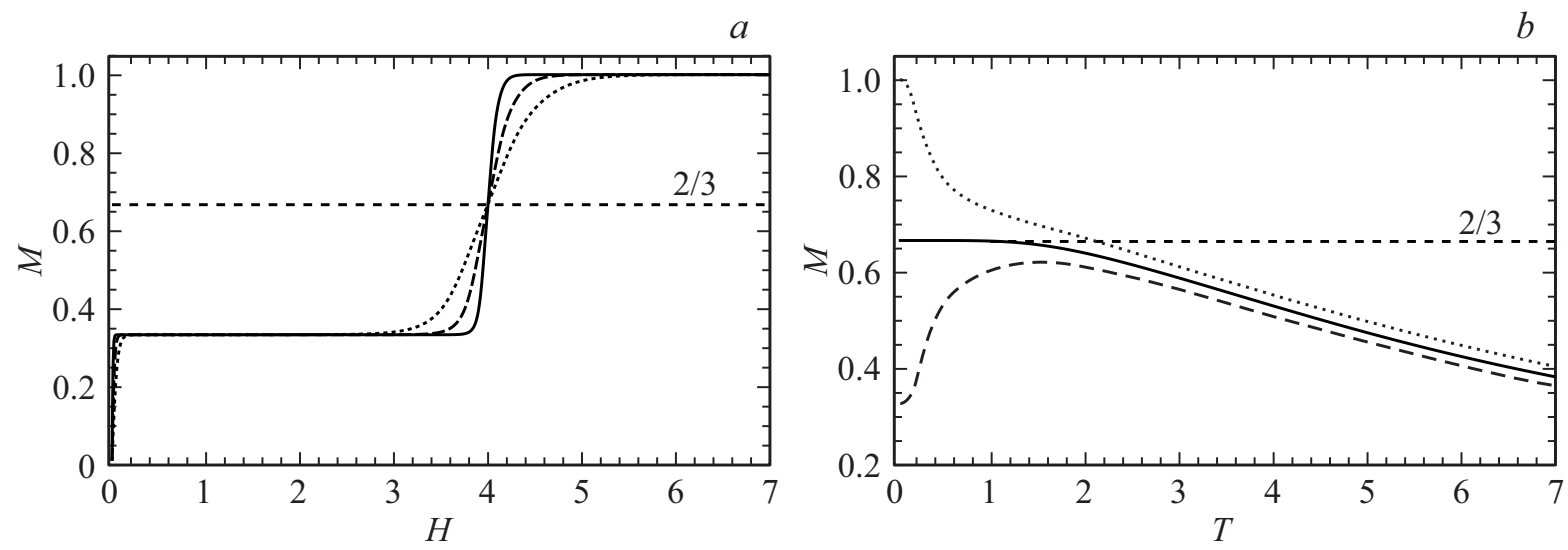

Рис. 5. Антиферромагнитная модель Изинга на однократно декорированной $\left(d_{x}=d_{y}=1\right)$ квадратной решетке $\left(J_{x}^{\prime}=J_{y}^{\prime}=-1\right)$ в магнитном поле: $(a)-$ намагниченность при $T=0.1$ (сплошная линия), $T=0.2$ (штриховая линия), $T=0.5$ (пунктирная линия); (b) $-h=3.8$ (штриховая линия), $h=4.0$ (сплошная линия), $h=4.2$ (пунктирная линия).

модействий $J_{x}$ и $J_{y}$ очевидна. Из численных расчетов следует вывод о том, что в антиферромагнитной и в ферро-антиферромагнитной модели магнитное поле постепенно подавляет температуру фазового перехода до нуля при поле, равном фрустрационному. Мы исследовали также влияние магнитного поля на температуру перехода в ферромагнетике. На рис. 4, а изображена теплоемкость для нескольких значений поля при $J_{x}=+1$ и $J_{y}=+1$, а на рис. $4, b$ намагниченность ферромагнетика в зависимости от поля.

Из рис. 4 следует, что фазовый переход в ферромагнетике, определяемый пиком логарифмической расходимости теплоемкости, существует только при $h=0$. При любом, сколь угодно малом поле, теплоемкость имеет плавный куполообразный вид без расходимости. Детальный анализ численных расчетов для модели Изинга на недекорированной квадратной решетке позволяет сделать выводы о том, что магнитное поле является фрустрирующим фактором. Другими словами, сколь угодно малое поле подавляет фазовый переход. Это объясняется тем, что в основном состоянии в отсутствие поля существует две конфигурации с равной внутренней энергией: все спины направлены вверх или все спины направлены вниз. Сколь угодно малое поле расщепляет эти энергии, так что наинизшей энергией начинает обладать конфигурация со спинами, направленными вдоль поля.

\section{4. Влияние поля на декорированной решетке в модели Изинга в отсутствие прямых взаимодействий}

Рассмотрим сначала квадратную решетку в отсутствие поля, однократно декорированную по двум направлениям (в формулах (1) и (2) это соответствует значениям $\left.d_{x}=d_{y}=1, h=0\right)$. Температура фазового перехода в частных случаях $\left|J_{x}^{\prime}\right|=\left|J_{y}^{\prime}\right|=1$ равна
$2 / \cosh ^{-1}(1+\sqrt{2}) \approx 1.308$ и не зависит от знаков взаимодействий $J_{x}^{\prime}$ и $J_{y}^{\prime}$, что имеет место и на недекорированной решетке $2 / \ln (1+\sqrt{2}) \approx 2.269$. Однако, поведение модели в поле совершенно различно в зависимости от знаков обменных взаимодействий $J_{x}^{\prime}$ и $J_{y}^{\prime}$.

Поведение ферромагнитной модели $\left(J_{x}^{\prime}=J_{y}^{\prime}=1\right)$ на рассматриваемой декорированной решетке подобно таковой на недекорированной решетке (рис. 4), за исключением только величины температуры фазового перехода. Даже бесконечно малое поле полностью подавляет фазовый переход.

Рассмотрим теперь поведение антиферромагнитной модели $\left(J_{x}^{\prime}=J_{y}^{\prime}=-1\right)$ на рассматриваемой однократно декорированной $\left(d_{x}=d_{y}=1\right)$ квадратной решетке в отсутствие прямых взаимодействий. Несмотря на общие с недекорированной антиферромагнитной решеткой черты: равенство фрустрационного поля $(h=4)$, одинаковое температурное поведение вблизи фрустрационного поля, следует отметить существенно отличные особенности декорированной решетки. В частности, несколько неожиданное ферромагнитное поведение намагниченности в полях от нулевого до фрустрационного. На языке конфигураций этот парадокс объясняется аналогичным образом.

В основном состоянии в отсутствие поля существует две конфигурации с равной внутренней энергией, а именно, в одной из них все декорационные спины направлены вверх и все нодальные спины направлены вниз; в другой конфигурации наоборот, все декорационные спины направлены вниз и все нодальные спины направлены вверх. Декорационных спинов в каждой конфигурации в два раза больше, чем нодальных, и поэтому энергия конфигурации с большим числом спинов, направленных вдоль поля, становится ниже энергии другой конфигурации.

Эта конфигурация с ростом магнитного поля остается превалирующей вплоть до значений фрустрационного поля $(h=4)$. Намагниченность плато на рис. 5, равная $1 / 3$, объясняется тем, что две трети спинов (деко- 

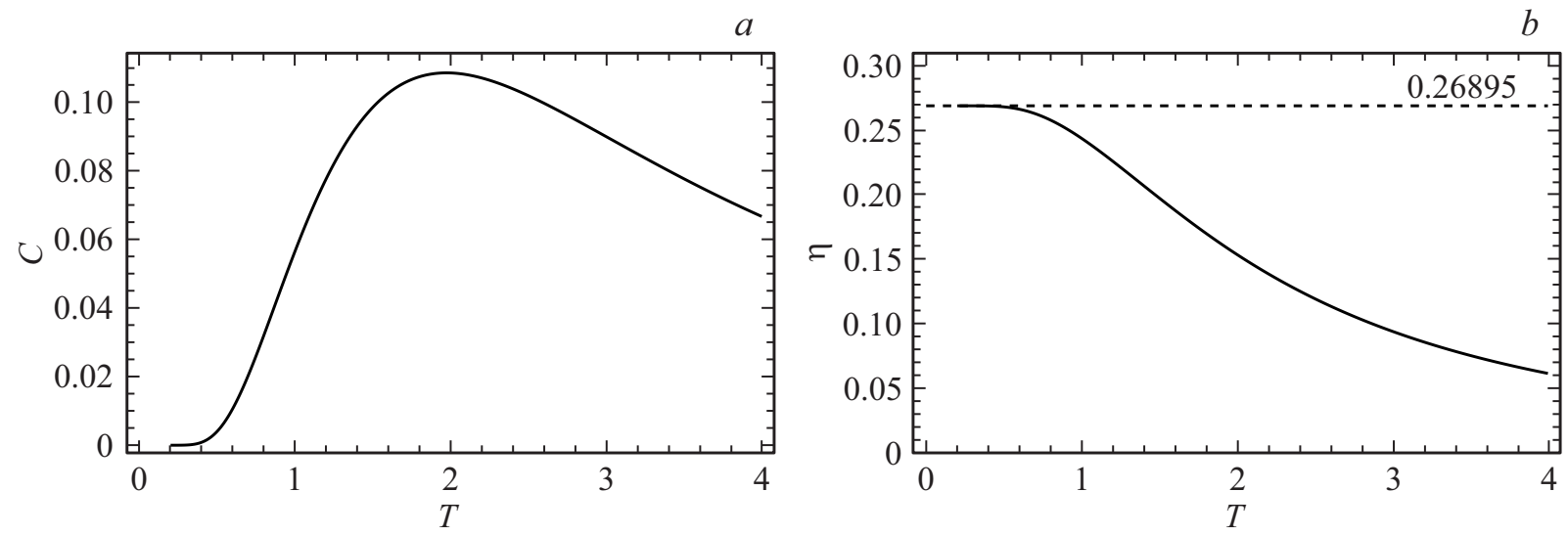

Рис. 6. Теплоемкость $(a)$ и параметр порядка $(b)$ декорированной решетки в присутствие прямых взаимодействий $\left(J_{x}=J_{y}=J_{x}^{\prime}=J_{y}^{\prime}=-1\right)$.
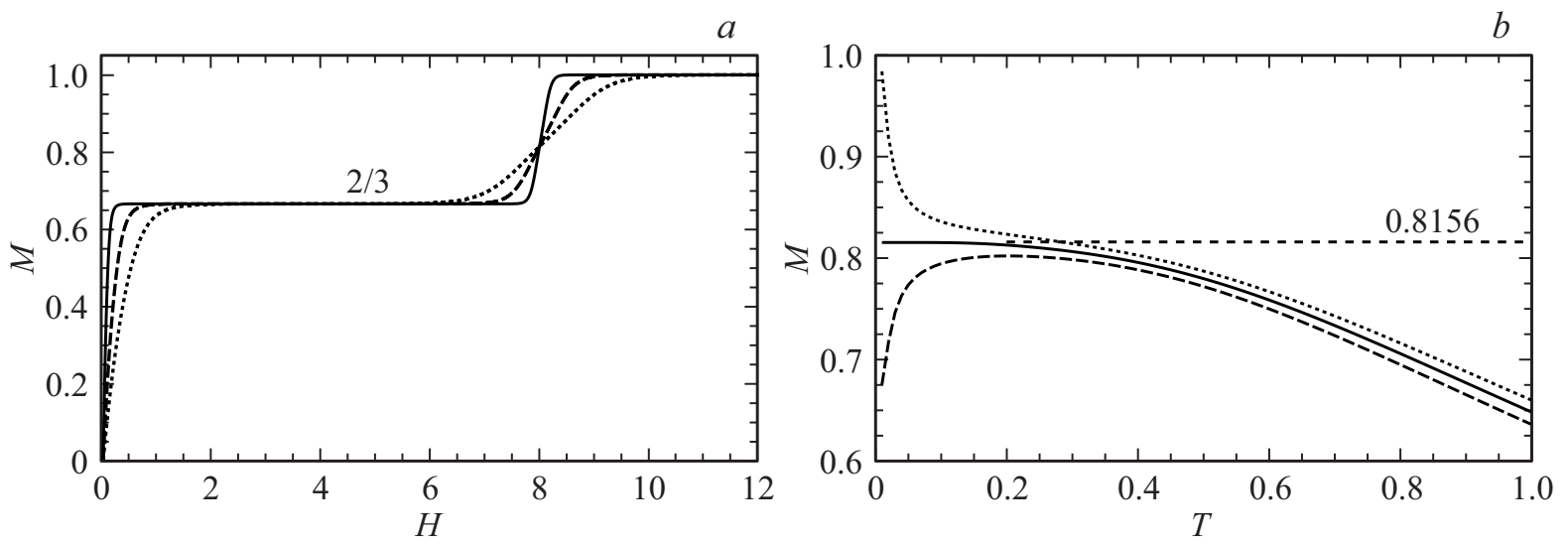

Рис. 7. Намагниченность антиферромагнитной декорированной решетки в присутствие прямых взаимодействий $\left(J_{x}=J_{y}=J_{x}^{\prime}=J_{y}^{\prime}=-1\right)$. Полевая зависимость $(a)$ : сплошная линия $-T=0.05$, штриховая линия $-T=0.2$, пунктирная линия $-T=0.5$; температурная зависимость $(b)$ : сплошная линия $-h=8.0$, штриховая линия $-h=7.75$, пунктирная линия $h=8.25$.

рационные) направлена вдоль поля, а одна треть спинов (нодальные) против поля.

Таким образом возникает эффект частичного упорядочения. На этом же рисунке нуль-температурное значение намагниченности во фрустрационном поле, равное $2 / 3$, свидетельствует о том, что при этом поле вклады в результирующую намагниченность от этой конфигурации и от конфигурации, в которой поле разворачивает уже все спины, равны.

Подобный сценарий наблюдается также в варианте ферро-антиферромагнитной модели $\left(J_{x}^{\prime}=1, J_{y}^{\prime}=-1\right)$. Главное отличие от предыдущего антиферромагнитного варианта $\left(J_{x}^{\prime}=J_{y}^{\prime}=-1\right)$ заключается в величине фрустрационного поля, равного двум. На языке конфигураций этот сценарий объясняется следующим образом.

В основном состоянии в отсутствие поля существует также две конфигурации с равной внутренней энергией. В каждой из них все спины (как нодальные, так и декорационные) вдоль любой выбранной оси $x$ сонаправлены (либо вверх, либо вниз). Все остальные декорационные спины направлены в противоположном направлении.
В результате число сонаправленных спинов в каждой из этих двух конфигураций также оказывается в два раза больше, так что высота плато также равна 1/3. Величина фрустрационного поля в данном варианте в два раза ниже, потому что для переворота всех спинов поле преодолевает только одно взаимодействие $J_{y}^{\prime}$ между осями $x$, а вдоль этих осей все спины уже выстроены ферромагнитно.

\section{5. Влияние поля на декорированной решетке в модели Изинга с учетом прямых взаимодействий}

Поведение ферромагнитной модели $\left(J_{x}=J_{y}=J_{x}^{\prime}=\right.$ $\left.=J_{y}^{\prime}=1\right)$ на рассматриваемой декорированной решетке в присутствие взаимодействий между нодальными спинами совершенно аналогично поведению на недекорированной решетке (рис. 4), за исключением только величины температуры фазового перехода, равной $2 / \ln (\sqrt{1+2 \sqrt{2}})$. Бесконечно малое поле также пол- 


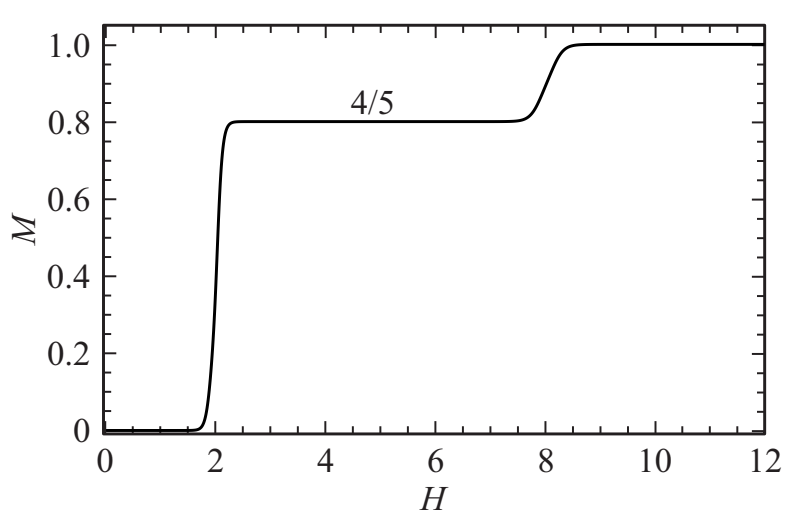

Рис. 8. Намагниченность дважды декорированной $(d=2)$ антиферромагнитной решетки $\left(J_{x}=J_{y}=J_{x}^{\prime}=J_{y}^{\prime}=-1\right)$.

ностью подавляет фазовый переход, существующий в отсутствие магнитного поля.

В полностью антиферромагнитной модели $\left(J_{x}=J_{y}=\right.$ $\left.=J_{x}^{\prime}=J_{y}^{\prime}=-1\right)$ на однократно декорированной решетке возникает совершенно новое явление, а именно, уже в отсутствие магнитного поля фазовый переход отсутствует из-за фрустраций, которые порождены конкуренцией прямых и декорационных взаимодействий. На рис. 6 представлена температурная эволюция теплоемкости (верхняя панель) и параметра порядка с нультемпературным значением, равным $\approx 0.2689$ (нижняя панель), что явно свидетельствует о наличии сильных фрустраций без какого-либо частичного упорядочения. Однако поведение намагниченности, изображенное на рис. 7, аналогично антиферромагнитной модели на декорированной решетке без прямых взаимодействий $\left(J_{x}^{\prime}=J_{y}^{\prime}=-1\right)$ (см. рис. 5). Влияния прямых взаимодействий заключаются в изменении величины фрустрационного поля, равного четырем вместо двух, нультемпературной намагниченности 0.8156 вместо $2 / 3$ и промежуточного плато с высотой $2 / 3$ вместо $1 / 3$. При некоторых других соотношениях параметров антиферромагнитных взаимодействий, например, $\left(J_{x}=J_{y}=J_{x}^{\prime}=-1, J_{y}^{\prime}=-0.5\right)$, фазовый переход возникает, но качественно поведение намагниченности сохраняется.

Декорирование с кратностью выше единичного вносит свои особенности во влияние магнитного поля термодинамические и магнитные свойства, в том числе и на фазовый переход. Например, в случае двукратного декорирования при таких же параметрах $\left(J_{x}=J_{y}=J_{x}^{\prime}=J_{y}^{\prime}=-1\right)$ намагниченность (рис. 8) возникает по антиферромагнитному сценарию, высота промежуточного плато декорированной равна $4 / 5$, фрустрационных полей два (2 и 8), причем фазовый переход при нулевом поле существует с точкой перехода, равной $T_{c}=2.5669$. В результате, как показывают расчеты теплоемкости, фазовый переход подавляется магнитным полем до нуля уже в первом фрустрационном поле, равном двум.

\section{6. Заключение}

В работе проведено исследование модели Изинга на недекорированной и декорированной квадратной решетке с разнообразными параметрами ферромагнитных и антиферромагнитных обменных взаимодействий во внешнем магнитном поле.

Подавление магнитным полем фазового перехода происходит всегда, при любых величинах и знаках всех обменных взаимодействий. Причем обнаружены два типа сценариев, что связано с поведением намагниченности на начальном этапе возникновения и роста магнитного поля. В первом случае, если намагниченность растет по антиферромагнитному типу, то есть, форма кривой на этом этапе вогнутая, то происходит уменьшение точки исходного (в отсутствие магнитного поля) фазового перехода до нуля при достижении первого фрустрационного поля, и при дальнейшем увеличении поля фазовый переход не возникает.

В противоположном случае, когда намагниченность растет по ферромагнитному типу, то есть, форма кривой на этом этапе выпуклая, то фазовый переход исчезает сразу же при включении поля, другими словами,сколь угодно малое поле полностью подавляет фазовый переход. Следует отметить парадоксальную особенность данного случая, заключающуюся в том, что намагниченность может расти по ферромагнитному типу, если даже все обменные параметры модели антиферромагнитные.

\section{Финансирование работы}

Работа выполнена в рамках государственного задания Минобрнауки России (тема Квант, № АААА-А18118020190095-4), при частичной поддержке Уральского Отделения РАН (проект № 18-2-2-11).

\section{Конфликт интересов}

Авторы заявляют, что у них нет конфликта интересов.

\section{Список литературы}

[1] I. Syôzi. Prog. Theor. Phys., 6, 306 (1951).

[2] Б.К. Вайнштейн. Современная кристаллография. Симметрия кристаллов. Методы структурной кристаллографии. Наука, М., 1979. Т. 1. 384 с.

[3] F. Kassan-Ogly, B. Filippov, A. Murtazaev, M. Ramazanov, M. Badiev. J. Magn. Magn. Mater. 324, 3418 (2012).

[4] F. Wang, D.P. Landau. Phys. Rev. Lett. 86, 2050 (2001).

[5] F. Wang, D.P. Landau. Phys. Rev. E 64, 056101 (2001).

6] L. Onsager. Phys. Rev. 65, 117 (1944).

[7] E. Müller-Hartmann, J. Zittartz. Z. Physik B 27, 261 (1977).

[8] F. Kassan-Ogly, A. Murtazaev, A. Zhuravlev, M. Ramazanov, A. Proshkin. J. Magn. Magn. Mater. 384, 247 (2015).

Редактор Ю.Э. Китаев 